\title{
Ramsey theory for layered semigroups
}

\author{
Jordan Mitchell Barrett \\ School of Mathematics and Statistics \\ Victoria University of Wellington \\ New Zealand \\ math@jmbarrett.nz
}

Submitted: Oct 11, 2020; Accepted: Apr 9, 2021; Published: Apr 23, 2021

(C) The author. Released under the CC BY license (International 4.0).

\begin{abstract}
We further develop the theory of layered semigroups, as introduced by Farah, Hindman and McLeod, providing a general framework to prove Ramsey statements about such a semigroup $S$. By nonstandard and topological arguments, we show Ramsey statements on $S$ are implied by the existence of "coherent" sequences in $S$. This framework allows us to formalise and prove many results in Ramsey theory, including Gowers' FIN $_{k}$ theorem, the Graham-Rothschild theorem, and Hindman's finite sums theorem. Other highlights include: a simple nonstandard proof of the Graham-Rothschild theorem for strong variable words; a nonstandard proof of Bergelson-Blass-Hindman's partition theorem for located variable words, using a result of Carlson, Hindman and Strauss; and a common generalisation of the latter result and Gowers' theorem, which can be proven in our framework.
\end{abstract}

Mathematics Subject Classifications: 05D10, 03H05, 22A20, 54J05, 54D80

\section{Introduction}

Ramsey theory mathematically studies to what extent regular configurations appear in disorder. A Ramsey-type result typically has the following form: for any finite colouring of some structure $\mathcal{M}$, we can find a monochromatic substructure $\mathcal{N} \subseteq M$ with certain properties. The structure $\mathcal{M}$ and required properties of $\mathcal{N}$ are what distinguish the various results. Commonly, the structure in question will be a semigroup. An early example is van der Waerden's theorem on monochromatic arithmetic progressions:

Theorem 1 (van der Waerden). For every $k \in \mathbb{N}$ and finite colouring of $\mathbb{N}$, there is $a, d \in \mathbb{N}$ such that the arithmetic progression $a, a+d, a+2 d, \ldots, a+(k-1) d$ is monochromatic. 
Here, the structure in question is the semigroup $(\mathbb{N},+)$. A later example, more in the style of the results of this paper, is the Hales-Jewett theorem about the word semigroup $A^{<\omega}$ over a finite alphabet $A$. We let $V=(A \cup\{x\})^{<\omega} \backslash A^{<\omega}$ be the set of variable words, words over $A$ which include the variable symbol $x$. Given $u \in V$ and $a \in A$, the (nonvariable) word $u[a]$ is formed by replacing each occurrence of $x$ in $u$ with $a$.

Theorem 2 (Hales-Jewett). For every finite colouring of $A^{<\omega}$, there is a variable word $u \in V$ such that $\{u[a]: a \in A\}$ is monochromatic.

Infinitary Ramsey theory received a boost in the 1970s with the advent of ultrafilter methods, as pioneered by Glazer in his proof of Hindman's finite sums theorem [5, Thm 10.3]. Given a semigroup $(S,+)$, we can naturally extend + to an operation $\oplus$ on the set $\beta S$ of ultrafilters on $S$. Furthermore, $\beta S$ admits a natural topology, making it a compact right-topological semigroup. The rich algebraic structure of $\beta S$ has powerful applications and consequences all throughout combinatorics [18]. More recently, nonstandard methods have also seen success in Ramsey theory [6], particularly in studying partition regularity of Diophantine equations [9, 10,3].

In [11], Farah, Hindman and McLeod introduced layered semigroups, as well as shifts and layered actions thereon. The motivation was to generalise partition results about certain spaces of variable words, such as Gowers' FIN $_{k}$ theorem, the Hales-Jewett theorem, and Bergelson, Blass and Hindman's theorem on located words. Layered semigroups were further explored by Lupini [20] and Farmaki-Negrepontis [13].

This paper should be considered a "spiritual successor" to [11]. We also work in the setting of layered semigroups, but we consider a different, much broader class of morphisms, called regressive maps. Working in this setting, we develop a general framework to prove partition theorems about a layered semigroup $S$, assuming only the existence of certain "coherent" sequences in $S$. This framework allows a general way to formulate and prove many fundamental results of Ramsey theory.

While [11] was phrased in the language of ultrafilters, we instead formalise our results using nonstandard analysis. We believe the nonstandard formulation is more intuitive, but our work has an equivalent translation in the setting of ultrafilters. §2.1 reviews the necessary concepts of nonstandard analysis, working in an internal superstructure model. Effectively, every object $M$ under consideration is assigned a nonstandard extension * $M$, such that the transfer principle holds $-M$ and * $M$ satisfy the same "elementary" properties.

Ellis' theory of compact semitopological semigroups (CSTSs) is also essential to our nonstandard study of Ramsey theory, and we discuss the topological prerequisites in $\S 2.2$. For a semigroup $S$, we define a topology on ${ }^{*} S$ such that ${ }^{*} S$ is "nearly" a CSTS. This gives us analogues of results in CSTS theory - particularly the Ellis-Numakura lemma guaranteeing the existence of idempotents, which are essential to our work.

In $\S 3$, we define layered semigroups $S$ - those which can be partitioned into countably many layers $S_{0}, S_{1}, \ldots$ so that $S_{0} \cup \cdots \cup S_{n}$ forms a semigroup, of which $S_{n}$ is an ideal. We see some examples which naturally occur in Ramsey theory, some of which are in fact partial, but adequate in a specified sense. $\S 4$ considers regressive maps on $S$ - semigroup 
homomorphisms $f: S \rightarrow S$ which map layers downwards, and don't separate or reorder them. Natural examples of maps on layered semigroups are generally regressive, hence this notion distills the essential Ramsey-theoretic properties of such maps.

In $\S 3.1$ and $\S 4.1$, nonstandard analysis comes in, as we consider sequences $\left(\alpha_{i}\right)$ of nonstandard elements where $\alpha_{n} \in{ }^{*} S_{n}$. Such a sequence is coherent if it is closed under all regressive maps under consideration, and Ramsey if $\alpha_{n}$ absorbs all $\alpha_{i}, i \leqslant n$ under the semigroup operation. We present the main mechanism for proving Ramsey statements in $\S 5$. If $\mathcal{F}$ is a collection of regressive maps on $S$, the framework is summarised diagrammatically below:

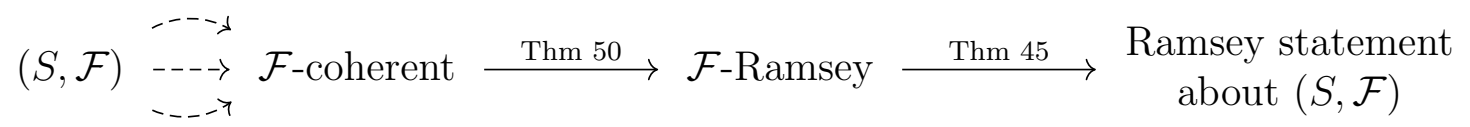

It is difficult to construct general arguments giving the implication $--\rightarrow$ above, without having to impose very strong conditions on $S$ and $\mathcal{F}$. Therefore, the construction of an $\mathcal{F}$-coherent will usually depend on the specific semigroup under consideration. The most general construction we give is Lemma 76, for "complete" subsemigroups of FIN ${ }^{A}$ this covers Gowers' theorem and Bergelson-Blass-Hindman's theorem on located variable words.

However, the other two implications work much more generally, only requiring weak, natural conditions on $S$ and $\mathcal{F}$. As a result, Ramsey statements on $(S, \mathcal{F})$ can be reduced to the existence of coherent sequences in $S$. Towards the end of the paper, we show how our general framework recovers many fundamental results in Ramsey theory, including

- Gowers' $\mathrm{FIN}_{k}$ theorem, and its generalisation due to Lupini (§6.1);

- The Graham-Rothschild parameter sets theorem ( $(6.2)$;

- The Galvin-Glazer theorem and Hindman's finite sums theorem (§6.3);

- An infinitary, multivariable generalisation of Bergelson, Blass and Hindman's partition theorem on located variable words $(\S 7)$.

Collectively, these theorems imply a variety of other Ramsey-type results, including Hindman's finite unions theorem, the Hales-Jewett theorem, and van der Waerden's theorem. In each case, we give elementary nonstandard constructions of coherent sequences, which is enough to imply the corresponding result via our framework. We also present a common generalisation of Gowers' theorem and the multivariable BergelsonBlass-Hindman theorem (and even the Milliken-Taylor theorem) in $\S 7.1$, which is provable using our framework. Again, an elementary nonstandard argument constructs an $\mathcal{F}$-coherent in this case.

Throughout, we let $\mathbb{N}=\{0,1,2, \ldots\}$ be the set of nonnegative integers. We may use interval notation, e.g. $[0,3],[1,7)$, and this should be interpreted in the natural numbers, i.e. $[n, m]=\{k \in \mathbb{N}: n \leqslant k \leqslant m\}$. This notation will later extend to nonstandard integers ${ }^{*} \mathbb{N}$, i.e. $[\xi, \zeta]=\{\alpha \in * \mathbb{N}: \xi \leqslant \alpha \leqslant \zeta\}$. 
In general, we will use uppercase Latin letters $A, B, \ldots, S, T, \ldots$ for sets and semigroups, lowercase Latin letters $s, t, \ldots$ for elements thereof, and lowercase Greek letters $\alpha, \beta, \ldots$ for elements of nonstandard extensions ${ }^{*} S$ of semigroups.

\section{Prerequisites}

\subsection{Nonstandard analysis}

The main results of this paper (in $\S 5$ ) will be proved using the tools of nonstandard analysis. Here, we give a basic overview of the concepts needed - for a more in-depth exposition of nonstandard methods and their applications to Ramsey theory, see [6]. All our work can alternatively be formulated using ultrafilter methods, as per [24, 18].

Effectively, we work inside a "universe" $\mathbb{V}$ which includes the semigroup $(S,+)$ under consideration, as well as subsets thereof and functions $f: S^{k} \rightarrow S^{m}$. This universe comes equipped with a star map $*: \mathbb{V} \rightarrow \mathbb{V}$, which assigns every object $M \in \mathbb{V}$ to its nonstandard extension ${ }^{*} M$, in such a way that the following properties hold:

Axioms 3 (Basic properties of the star map).

(i) For a set $A,{ }^{*} A$ is also a set, and ${ }^{\sigma} A \subseteq{ }^{*} A$, where ${ }^{\sigma} A:=\left\{{ }^{*} x: x \in A\right\}$. This containment is strict iff $A$ is infinite;

(ii) If $A, B$ are sets such that $A \subseteq B$, then ${ }^{*} A \subseteq{ }^{*} B$;

(iii) For a set $A$ and $k \in \mathbb{N}$, we have ${ }^{*}\left(A^{k}\right)=\left({ }^{*} A\right)^{k}$;

(iv) If $f$ is a function $A \rightarrow B$, then ${ }^{*} f$ is a function ${ }^{*} A \rightarrow{ }^{*} B$;

(v) If $f: A \rightarrow B$ and $x \in A$, then $\left({ }^{*} f\right)(x)=f(x)$;

(vi) If $f: A \rightarrow B$ and $x \in A$, then $\left({ }^{*} f\right)\left({ }^{*} x\right)={ }^{*}(f(x))$;

(vii) If $s \in S$, then ${ }^{*} s=s$;

(viii) If $n \in \mathbb{N}$, then ${ }^{*} n=n$.

The star map also satisfies the following key principle:

Axiom 4 (Transfer principle). For any elementary ${ }^{1}$ formula $\varphi\left(x_{1}, \ldots, x_{n}\right)$ and objects $M_{1}, \ldots, M_{n} \in \mathbb{V}$, we have

$$
\varphi\left(M_{1}, \ldots, M_{n}\right) \text { holds } \Longleftrightarrow \varphi\left({ }^{*} M_{1}, \ldots,{ }^{*} M_{n}\right) \text { holds }
$$

\footnotetext{
${ }^{1} \mathrm{~A}$ formula is elementary if all the quantifiers are bounded, i.e. of the form $Q x \in y$ for objects $x, y \in \mathbb{V}$. All logical formulae which we consider will be elementary.
} 
For a rigorous construction satisfying Axioms 3 and 4, see [6]. Here, we will take on faith that such a structure does exist.

The semigroup operation + can be considered as a function $+: S^{2} \rightarrow S$, thus we get a natural extension ${ }^{*}+$ of this operation to ${ }^{*} S$. Abusing notation, we will use + to denote both the original operation and its nonstandard extension - this is somewhat justified by Axiom 3.(v). We will generally do the same for functions $f: S^{k} \rightarrow S^{m}$.

A peculiarity of our approach will be that we may iterate the star map, to obtain nonstandard extensions of nonstandard extensions, and so on. In this way, we get objects $M,{ }^{*} M,{ }^{* *} M, \ldots$. We will use ${ }^{n *} M$ to denote the $n$-fold nonstandard extension of an object $M \in \mathbb{V}$. Axioms 3 and 4 also hold when the objects under consideration are themselves nonstandard.

Remark 5. In general, the simplifying assumptions made in Axioms 3.(vii) and 3.(viii) cannot be extended to elements of ${ }^{*} S, * \mathbb{N}$ or higher in the nonstandard hierarchy. As an example, $\mathbb{N}$ is an initial segment of $* \mathbb{N}\left[6\right.$, Prop 2.27], so by transfer, ${ }^{*} \mathbb{N}$ is an initial segment of ${ }^{* *} \mathbb{N}$. Now, if we take $\xi \in *^{*} \mathbb{N} \backslash \mathbb{N}$, we have ${ }^{*} \xi \in{ }^{* *} \mathbb{N} \backslash * \mathbb{N}$ by transfer. It follows that $\xi<{ }^{*} \xi \Longrightarrow \xi \neq *^{*} \xi$.

\section{$2.2 u$-semigroups}

Here, we develop some further notions that prove essential in the study of Ramsey semigroups. These are mostly based on the theory of compact semitopological semigroups (see $[24, \S 2]$ ), as developed by Ellis and others. An example is given by $\beta S$, the set of ultrafilters on $S$, whose topological and algebraic structure is well-studied [18, 24]. $\beta S$ is also homeomorphic to the Stone-Čech compactification of $S$ with the discrete topology. The following map allows us to transport this structure to ${ }^{*} S$.

Definition 6. Elements $\alpha \in{ }^{*} S$ generate ultrafilters on $S$ via the ultrafilter map:

$$
\alpha \mapsto \mathcal{U}_{\alpha}=\left\{A \subseteq S: \alpha \in^{*} A\right\}
$$

Two elements $\alpha, \beta$ are $u$-equivalent (denoted $\alpha \sim \beta$ ) if $\mathcal{U}_{\alpha}=\mathcal{U}_{\beta}$.

Proposition 7. $\sim$ is an equivalence relation on ${ }^{*} S$.

The relation $\sim$ on ${ }^{*} S$ was first considered by Di Nasso in [7], and has seen extensive combinatorial and Ramsey-theoretic applications in $[6,9,10,3]$. Some of the key properties are summarised below.

Proposition $8([7,6])$.

(i) If $\alpha \in{ }^{*} S, s \in S$, then $\alpha \sim s$ if and only if $\alpha=s$.

(ii) For any function $f: S \rightarrow S$, if $\alpha \sim \beta \in{ }^{*} S$ then $f(\alpha) \sim f(\beta)$.

(iii) For any function $f: S \rightarrow S$, if $\alpha \in{ }^{*} S$ is such that $f(\alpha) \sim \alpha$, then $f(\alpha)=\alpha$.

(iv) For any $\alpha, \alpha^{\prime}, \beta, \beta^{\prime} \in{ }^{*} S$, if $\alpha \sim \alpha^{\prime}$ and $\beta \sim \beta^{\prime}$, then $\alpha+{ }^{*} \beta \sim \alpha^{\prime}+{ }^{*} \beta^{\prime}$. 
(v) For any $\alpha \in{ }^{*} S, \alpha{ }^{*} \alpha$.

There is a natural way to define a topology on ${ }^{*} S$ as follows:

Definition 9. For any semigroup $S$, equip ${ }^{*} S$ with the $u$-topology - that generated by the basic open sets ${ }^{*} A$ for $A \subseteq S$. We say ${ }^{*} S$ is a compact $u$-semigroup, i.e.

(i) ${ }^{*} S$ is compact;

(ii) For any $\alpha, \beta \in S$, there exists $\gamma \in S$ such that $\gamma \sim \alpha+{ }^{*} \beta$;

(iii) The map $\alpha \mapsto \alpha+{ }^{*} \beta$ is continuous.

Proposition 10. ${ }^{*} S / \sim$ is Hausdorff, i.e. two elements $\alpha, \beta \in{ }^{*} S$ are inseparable by disjoint open sets exactly when $\alpha \sim \beta$.

Corollary 11. For continuous functions $f, g:{ }^{*} S \rightarrow{ }^{*} S$, the set $\left\{\alpha \in{ }^{*} S: f(\alpha) \sim g(\alpha)\right\}$ is closed.

Proposition 12. For any function $f: S \rightarrow S$, its nonstandard extension ${ }^{*} f:{ }^{*} S \rightarrow{ }^{*} S$ is continuous with respect to the $u$-topology on ${ }^{*} S$.

Idempotent ultrafilters are key to most applications of infinitary methods in Ramsey theory - their existence follows from the Ellis-Numakura lemma. Throughout this paper, we will use a similar notion of idempotence for elements of ${ }^{*} S$.

Definition 13. Suppose $(S,+)$ is a semigroup. We say $\alpha \in{ }^{*} S$ is $u$-idempotent if $\alpha+{ }^{*} \alpha \sim$ $\alpha$.

Lemma 14 (Ellis-Numakura). If $T \subseteq{ }^{*} S$ is a closed u-subsemigroup, then $T$ contains a $u$-idempotent element.

Often, we will need a strengthening of Lemma 14, as follows.

Definition 15. Define a relation $\preccurlyeq$ on ${ }^{*} S$ by

$$
\alpha \preccurlyeq \beta \Longleftrightarrow \alpha+{ }^{*} \beta \sim \beta+{ }^{*} \alpha \sim \alpha
$$

$\preccurlyeq$ is a partial order (up to $u$-equivalence) on the $u$-idempotents of ${ }^{*} S$.

Corollary 16 ([24, Lemma 2.3]). Any closed u-subsemigroup of ${ }^{*} S$ contains a $\preccurlyeq$-minimal u-idempotent element. 


\section{$3 \quad$ Layered semigroups}

Our results concern the framework of layered semigroups, as introduced by Farah, Hindman and McLeod in [11]. We will work with the following adaptation of their definition:

Definition 17. A layered semigroup is a (total) semigroup $S$, with a layering map $\ell$ : $S \rightarrow \mathbb{N}$ such that for all $s, t \in S, \ell(s+t)=\max \{\ell(s), \ell(t)\}$.

The map $\ell$ splits $S$ into layers $S_{n}=\ell^{-1}(n)$ for each $n \in$ range $(\ell)$. Without loss of generality, we will suppose ${ }^{2}$ that range $(\ell)$ is an initial segment of $\mathbb{N}$. We will encounter situations where range $(\ell)$ is finite (i.e. our semigroup has finitely many layers), but also cases when range $(\ell)=\mathbb{N}$ (i.e. our semigroup has infinitely many layers. In theory, we could allow range $(\ell)=\delta$ for ordinals $\delta>\omega$, but we will not pursue such generalisations here.

Definition 17 is equivalent to the following, which is more in the style of Farah, Hindman and McLeod's original definition:

Proposition 18. A pair $(S, \ell: S \rightarrow \mathbb{N})$ form a layered semigroup if and only if:

(i) $S_{\leqslant n}:=\ell^{-1}(\{0, \ldots, n\})$ is a subsemigroup of $S$;

(ii) $S_{n}=\ell^{-1}(n)$ is a two-sided ideal of $S_{\leqslant n}$.

Remark 19. Farah, Hindman and McLeod's original definition in [11] is given by only allowing finitely many layers, and further positing that $S_{0}=\{e\}$, where $e$ is a two-sided identity for any element of $S$. We have relaxed both conditions, since we will encounter layered semigroups for which neither holds.

To illustrate Definition 17, we present some examples of layered semigroups which naturally arise in Ramsey theory, and which will be relevant later in this paper.

Example 20. For each $i$, let $M_{i}$ be a monoid (semigroup with identity $e_{i}$ ), such that only the identity has an inverse. Let $S$ be the set of tuples $\left(m_{0}, m_{1}, m_{2}, \ldots\right) \in \prod_{i=0}^{\infty} M_{i}$ with finite support (i.e. $m_{n} \neq e_{n}$ for finitely many $n$ ). Then, $S$ is a layered semigroup under pointwise operations, and the layering $\operatorname{map} \ell\left[\left(m_{i}\right)\right]=\min \left\{k: \forall i \geqslant k m_{i} \neq e_{i}\right\}$.

Example 21. Fix a finite alphabet $A$. For $k \in \mathbb{N}$, a $k$-parameter word is an element of $\left(A \cup\left\{x_{1}, x_{2}, \ldots, x_{k}\right\}\right)^{<\omega}$ such that all the variables $x_{1}, x_{2}, \ldots, x_{k}$ appear, and their first appearances are in increasing order. Let $W_{k}$ be the set of all $k$-parameter words. ${ }^{3}$ Then, $W=\bigcup_{k=0}^{\infty} W_{k}$ is a layered semigroup ${ }^{4}$ under concatenation, called the Graham-Rothschild semigroup.

We will also consider partial semigroups - those for which the operation is not always defined. To exclude trivial cases, such as when the operation is never defined, we have the following notion of adequacy for a partial semigroup.

\footnotetext{
${ }^{2}$ By shifting down values of $\ell$ as required.

${ }^{3}$ So $W_{0}$ is simply $A^{<\omega}$, the set of all words over $A$.

${ }^{4}$ The layering map $\ell: W \rightarrow \mathbb{N}$ here maps every parameter word to the number of variables it contains.
} 
Definition 22. A partial semigroup $S$ is adequate [11, 18, Defn 1.15.6] or directed [24] if, for any finite subset $F \subseteq S$, there exists $y$ such that $x+y$ is defined for all $x \in F$.

We now generalise Definition 17 to the case of partial semigroups. However, it is not enough for just $S$ to be adequate - we need each layer to be adequate also.

Definition 23. An adequate partial layered semigroup is a partial semigroup $S$, with a layering map $\ell: S \rightarrow \mathbb{N}$ such that:

(i) For all $s, t \in S$ such that $s+t$ is defined, $\ell(s+t)=\max \{\ell(s), \ell(t)\}$;

(ii) For all $s_{1}, \ldots, s_{n} \in S$ with $\ell\left(s_{1}\right)=\cdots=\ell\left(s_{n}\right)$, there is $t \in S$ such that $\ell(t)=\ell\left(s_{i}\right)$ and $s_{i}+t$ is defined for all $i \leqslant n$.

Example 24. Let FIN be the set of all functions $f: \mathbb{N} \rightarrow \mathbb{N}$ with finite support, i.e. $\operatorname{supp}(f):=\{n \in \mathbb{N}: f(n) \neq 0\}$ is finite. For $f, g \in$ FIN, we define $f+g$ pointwise iff the pointwise product $f(n) g(n)=0$ for all $n \in \mathbb{N} .^{5}$ Then, FIN is an adequate partial layered semigroup under the layering map $\ell(f)=\max (\operatorname{range}(f))$, called the Gowers semigroup.

Example 25. Later, we will consider nonstandard extensions of these semigroups. We use transfer to deduce what these extensions look like. For example, ${ }^{*} \mathrm{FIN}_{k}$ will consist ${ }^{6}$ of functions $\varphi:{ }^{*} \mathbb{N} \rightarrow[0, k]$ with hyperfinite support $\left(\right.$ i.e. $\operatorname{supp}(\varphi) \subseteq[0, \xi]$ for some $\left.\xi \in{ }^{*} \mathbb{N}\right)$ and having $\max (\operatorname{range}(\varphi))=k$. This is clear from writing the definition of $\mathrm{FIN}_{k}$ as an elementary formula, and applying transfer.

Remark 26. In general, $\bigcup_{i<\delta}{ }^{*} S_{i} \subseteq{ }^{*} S$, but this containment may be strict when $S$ has infinitely many layers. For example, in the Graham-Rothschild semigroup $W$ of Example $21,{ }^{*} W$ consists of parameter words having hyperfinite length ${ }^{7} \xi$ and $\zeta$-many variables for some $\xi, \zeta \in{ }^{*} \mathbb{N}$. In contrast, $\bigcup_{i=0}^{\infty}{ }^{*} W_{i}$ is the subset of ${ }^{*} W$ consisting of words with only finitely many variables. We will never need to consider all of ${ }^{*} S$; just the ${ }^{*} S_{i}$ will be enough for our purposes.

\subsection{The $*$-product $\Pi_{S}$}

We will consider sequences $\left(\alpha_{i}\right)$ of nonstandard elements, where each $\ell\left(\alpha_{i}\right)=i$. For notational convenience, we define the following:

Definition 27. Given a total layered semigroup $S$, define

$$
\Pi_{S}:=\prod_{i<\delta}^{*} S_{i}
$$

where $\delta$ is the number of layers. If it is clear what layered semigroup we are referring to, we may just use the notation $\Pi$.

\footnotetext{
${ }^{5}$ Equivalently, if $f$ and $g$ have disjoint supports.

${ }^{6}$ Not all such functions are in ${ }^{*} \mathrm{FIN}_{k}$ - only those that are internal. [6, $\left.\S 2.5\right]$ gives a good overview of internal/external objects. An understanding of these will not be necessary in this paper.

${ }^{7}$ This is clear by considering a $k$-parameter word $w \in W$ as a function $w:[1, n] \rightarrow A \cup\left\{x_{1}, x_{2}, \ldots, x_{k}\right\}$ for some $n \in \mathbb{N}$, and applying transfer.
} 
We now generalise Definition 27 to adequate partial semigroups.

Definition 28. For a subsemigroup $A \subseteq S$, we define

$$
{ }^{\gamma} A:=\left\{\alpha \in{ }^{*} A: x+\alpha \text { is defined for all } x \in A\right\}
$$

Example 29. Let FIN be the Gowers semigroup of Example 24. Then, for $k \in \mathbb{N},{ }^{\gamma} \mathrm{FIN}_{k}$ consists of the cofinite functions $\varphi \in{ }^{*} \mathrm{FIN}_{k}$ - those whose supports are disjoint from $\mathbb{N}$.

Remark 30. In the case of partial adequate semigroups $S$, generally the whole of $\beta S$ is not considered, but only a special subset, which is notated $\gamma S[24]$ or $\delta S[18,11]$. For each $A \subseteq S,{ }^{\gamma} A$ is the preimage of $\gamma A \subseteq \beta A$ under the ultrafilter map (Definition 6), hence the notation.

The sets ${ }^{\gamma} A$ are useful because they have the following property:

Proposition 31. If $\alpha \in{ }^{*} A$ and $\beta \in{ }^{\gamma} A$, then $\alpha+{ }^{*} \beta$ is always defined.

Proof. Since $\beta \in{ }^{\gamma} A$, the elementary formula

$$
\varphi\left(A,{ }^{*} A, \beta\right)=\forall x \in A \quad \exists y \in{ }^{*} A \quad x+\beta=y
$$

holds, so by transfer,

$$
\varphi\left({ }^{*} A,{ }^{* *} A,{ }^{*} \beta\right)=\forall x \in{ }^{*} A \quad \exists y \in{ }^{* *} A \quad x+{ }^{*} \beta=y
$$

also holds. Letting $x=\alpha$ gives the result.

Proposition 32. If $A \subseteq S$ is adequate, then ${ }^{\gamma} A$ is nonempty.

Proof. For each $s \in A$, let $K_{s}=\{y \in A: s+y$ is defined $\}$. Then we have

$$
{ }^{\gamma} A=\bigcap_{s \in A}{ }^{*} K_{s}
$$

By adequacy, each $K_{s}$ is nonempty, so each ${ }^{*} K_{s}$ is nonempty, and closed in the $u$-topology on $A$. The collection $\left\{{ }^{*} K_{s}: s \in A\right\}$ has the finite intersection property, since for any finite $F \subseteq A$ :

$$
\bigcap_{s \in F}{ }^{*} K_{s}=*\left[\bigcap_{s \in F} K_{s}\right]
$$

which is nonempty by adequacy of $A$. The result follows by compactness of ${ }^{*} A$ (it is a closed subset of the compact space ${ }^{*} S$ ).

Definition 33. Given an adequate layered semigroup $S$, define

$$
\Pi_{S}:=\prod_{i<\delta}{ }^{\gamma} S_{i}
$$

where $\delta$ is the number of layers. If it is clear what layered semigroup we are referring to, we may just use the notation $\Pi$.

Notice that if $S$ is total, then ${ }^{\gamma} A={ }^{*} A$ for any $A \subseteq S$, so Definitions 27 and 33 coincide. Proposition 34. For any layered semigroup $S, \Pi_{S}$ is a compact u-semigroup, with the product topology and operation defined componentwise. 


\section{Regressive maps}

Our Ramsey-type results will be in the context of certain functions acting on layered semigroups. We will require our functions $f: S \rightarrow S$ to have the following properties.

Definition 35. A regressive map is a function $f: S \rightarrow S$ such that for all $s, t \in S$ :

(i) $f(s t)=f(s) f(t)$, i.e. $f$ is a semigroup homomorphism;

(ii) $\ell(f(s)) \leqslant \ell(s)$;

(iii) $\ell(s) \leqslant \ell(t) \Longrightarrow \ell(f(s)) \leqslant \ell(f(t))$;

(iv) $|\ell(f(s))-\ell(f(t))| \leqslant|\ell(s)-\ell(t)|$

Remark 36. Property (i) seems natural, since we are dealing with semigroups. The "layering" of our semigroups motivates property (ii). More specifically, in $\S 5$, we will inductively construct "coherent" sequences $\alpha^{(0)} \in{ }^{\gamma} S_{0}, \alpha^{(1)} \in{ }^{\gamma} S_{1}, \ldots$ which are closed under all the functions $f$ under consideration. Property (ii) makes such a construction tractable, since we can build each $\alpha^{(k)}$ based only on the $\alpha^{(j)}$ for $j<k$.

Properties (iii) and (iv) are harder to motivate, but they are essential to the proof of Lemma 49, which plays a big part in the proof of Theorem 50. We will see below that many natural examples of maps on layered semigroups satisfy these two properties.

We will consider sequences which are "well-behaved" with respect to a collection $\mathcal{F}$ of regressive maps on $S$. Generally, $\mathcal{F}$ will be closed under composition-however, this is not required for our arguments to work. To make effective use of the transfer principle, we do require the following:

Definition 37. Let $\mathcal{F}$ be a collection of regressive maps $f: S \rightarrow S$. $\mathcal{F}$ is locally finite (l.f.) if for all $i \in \mathbb{N}$, the set $\mathcal{F}_{i}=\left\{\left.f\right|_{S_{\leqslant i}}: f \in \mathcal{F}\right\}$ is finite.

Example 38. Let $W$ be the Graham-Rothschild semigroup of Example 21. Now, we consider infinite parameter words $\tilde{w}$ - elements of $\left(A \cup\left\{x_{1}, x_{2}, \ldots\right\}\right)^{\omega}$ such that all $x_{i}$ appear, with their initial appearances in increasing order.

Every such $\tilde{w}$ defines a function $W \rightarrow W, u \mapsto u[\tilde{w}]$, called the substitution map, by replacing each occurrence of $x_{i}$ in $u$ with the $i$ th character in $\tilde{w}$. Each substitution map is a regressive map, and the collection $\mathcal{F}$ of all such maps is locally finite, and closed under composition.

Example 39. Let FIN be the Gowers semigroup of Example 24. Every $F: \mathbb{N} \rightarrow \mathbb{N}$ induces a map $\tilde{F}:$ FIN $\rightarrow$ FIN by composition, i.e. $\tilde{F}(f)=F \circ f$. When $F$ is a nondecreasing surjection, $\tilde{F}$ is a regressive map, and the collection $\mathcal{F}$ of all such maps is locally finite, and closed under composition. These maps, first considered in [2], are called (generalised) tetris operations. 


\subsection{Special sequences in $\Pi_{S}$}

Throughout this section, fix a layered semigroup $S$ and a locally finite collection of regressive maps $\mathcal{F}$ on $S$. Essential to the proof of Theorem 50 is the following notion of coherence for elements of $\Pi_{S}$.

Definition 40. An element $\left(\alpha_{i}\right)_{i<\delta} \in \Pi_{S}$ is $\mathcal{F}$-coherent if for all $f \in \mathcal{F}$ and $j<\delta$, we have $f\left(\alpha_{j}\right) \sim \alpha_{k}$ for some ${ }^{8} k \leqslant j$.

Intuitively, $\left(\alpha_{i}\right)$ is $\mathcal{F}$-coherent if it is closed under all functions $f \in \mathcal{F}$. Definition 40 doesn't say anything about the product of elements in $\left(\alpha_{i}\right)$, so a stronger notion of coherence is needed to draw conclusions about products.

Definition 41. An $\mathcal{F}$-coherent element $\left(\alpha_{i}\right)_{i<\delta} \in \Pi_{S}$ is $\mathcal{F}$-Ramsey if, for all $i \leqslant j<\delta$, $\alpha_{i} \succcurlyeq \alpha_{j} \cdot{ }^{9}$ We say $S$ itself is $\mathcal{F}$-Ramsey if $\Pi_{S}$ contains an $\mathcal{F}$-Ramsey element.

Remark 42. Sequences of ultrafilters satisfying the analogue of Definition 41 (as well as being $\preccurlyeq$-minimal) are termed reductive in [19].

\section{Main results}

Throughout this section, fix a layered semigroup $S$ and a locally finite collection of regressive maps $\mathcal{F}$ on $S$. The existence of an $\mathcal{F}$-Ramsey in $\Pi_{S}$ is a powerful statement-it implies that general Ramsey statements (Theorems 45 and 46) are true of our layered semigroup $S$. When put into context, these general statements reduce to familiar results of Ramsey theory. Before proving these statements, we need the following concept:

Definition 43. A sequence $\left(x_{i}\right) \subseteq S$ is called a block sequence $[14,20]$ or basic sequence $\left[24\right.$, Thm 2.20] if, for any $n_{0}<\cdots<n_{\ell-1}$ and $f_{0}, \ldots, f_{\ell-1} \in \mathcal{F}, f_{0}\left(x_{n_{0}}\right)+\cdots+f_{\ell-1}\left(x_{n_{\ell-1}}\right)$ is defined.

Remark 44. The above definition has content only when $S$ is partial - every sequence in a total semigroup is a block sequence.

Theorem 45. Suppose $S$ is $\mathcal{F}$-Ramsey with $\delta$ layers, and $n<\delta$. Then, for any finite colouring of $S$, there exists a block sequence $\left(x_{i}\right)_{i=1}^{\infty} \subseteq S_{n}$ such that for every $k \leqslant n$, the following set is monochromatic:

$$
S_{k} \cap\left\{f_{1}\left(x_{n_{1}}\right)+\cdots+f_{\ell}\left(x_{n_{\ell}}\right): 1 \leqslant n_{1}<\cdots<n_{\ell}, f_{1}, \ldots, f_{\ell} \in \mathcal{F}\right\}
$$

We sketch the proof. Note that for an $\mathcal{F}$-Ramsey $\left(\alpha_{i}\right)_{i<\delta} \in \Pi$, the constant sequence $\left(\alpha_{n}, \alpha_{n}, \alpha_{n}, \ldots\right)$ effectively satisfies the conclusion of the theorem, except that it is in ${ }^{\gamma} S_{n}$ rather than $S_{n}$. Given the existence of $\alpha_{n}$, we repeatedly apply transfer to deduce the existence of each $x_{i}$.

\footnotetext{
${ }^{8}$ Such a $k$ is uniquely defined.

${ }^{9}$ For the case $i=j$, this implies $\alpha_{i}$ is $u$-idempotent.
} 
Proof of Theorem 45. Fix an $\mathcal{F}$-Ramsey sequence $\left(\alpha_{i}\right)_{i<\delta} \in \Pi$. For each $i<\delta$, let $A_{i}=$ $\left\{x \in S_{i}: c(x)=c\left(\alpha_{i}\right)\right\}$, and $A=\bigcup_{i<\delta} A_{i}$.

$\alpha_{n}$ witnesses that the sentence ${ }^{10}$

$$
\exists \tau \in{ }^{*} S_{n}: \bigwedge_{f_{1}, f_{2} \in \mathcal{F}_{n}}\left(f_{1}(\tau) \in{ }^{*} A \wedge f_{1}(\tau)+{ }^{*} f_{2}\left(\alpha_{n}\right) \text { is defined and in }{ }^{* *} A\right)
$$

is true, so by transfer, there exists $x_{1} \in S_{n}$ such that, for all $f_{1}, f_{2} \in \mathcal{F}, f_{1}\left(x_{1}\right) \in A$, and $f_{1}\left(x_{1}\right)+f_{2}\left(\alpha_{n}\right)$ is defined and in ${ }^{*} A$.

We also have $f_{1}\left(x_{1}\right)+f_{2}\left(\alpha_{n}\right)+{ }^{*} f_{3}\left(\alpha_{n}\right) \in{ }^{* *} A$ for any choice of $f_{1}, f_{2}, f_{3} \in \mathcal{F}$. This is because $f_{2}\left(\alpha_{n}\right) \sim \alpha_{i}$ and $f_{3}\left(\alpha_{n}\right) \sim \alpha_{j}$ for some $i, j \leqslant n$, so either $f_{2}\left(\alpha_{n}\right)+{ }^{*} f_{3}\left(\alpha_{n}\right) \sim \alpha_{i} \sim$ $f_{2}\left(\alpha_{n}\right)$, or $f_{2}\left(\alpha_{n}\right)+{ }^{*} f_{3}\left(\alpha_{n}\right) \sim \alpha_{j} \sim f_{3}\left(\alpha_{n}\right)$. Hence, $\alpha_{n}$ witnesses the truth of

$$
\begin{aligned}
\exists \tau \in{ }^{*} S_{n}: \bigwedge_{f_{1}, f_{2}, f_{3} \in \mathcal{F}_{n}}\left(f_{1}(\tau)\right. & \in{ }^{*} A \wedge f_{1}(\tau)+{ }^{*} f_{2}\left(\alpha_{n}\right) \text { is defined and in }{ }^{* *} A \\
& \wedge f_{1}\left(x_{1}\right)+f_{2}(\tau) \text { is defined and in }{ }^{*} A \\
& \left.\wedge f_{1}\left(x_{1}\right)+f_{2}(\tau)+{ }^{*} f_{3}\left(\alpha_{n}\right) \text { is defined and in }{ }^{* *} A\right)
\end{aligned}
$$

whence by transfer, we get $x_{2} \in S_{n}$ with similar properties.

In general, suppose we have defined $x_{1}, \ldots, x_{j-1} \in S_{n}$ such that for all $\ell<j$, all $n_{1}<\cdots<n_{\ell}<j$, and all $f_{1}, \ldots, f_{\ell}, f \in \mathcal{F}$, we have

$$
\begin{aligned}
& f_{1}\left(x_{n_{1}}\right)+\cdots+f_{\ell}\left(x_{n_{\ell}}\right) \text { is defined and in } A \\
& f_{1}\left(x_{n_{1}}\right)+\cdots+f_{\ell}\left(x_{n_{\ell}}\right)+f\left(\alpha_{n}\right) \text { is defined and in }{ }^{*} A
\end{aligned}
$$

Then, by a similar argument to before, it is also true that $f_{1}\left(x_{n_{1}}\right)+\cdots+f_{\ell}\left(x_{n_{\ell}}\right)+f\left(\alpha_{n}\right)+$ ${ }^{*} f^{\prime}\left(\alpha_{n}\right)$ is defined and in ${ }^{* *} A$ for any choice of $f^{\prime} \in \mathcal{F}$. Thus, $\alpha_{n}$ witnesses the truth of

$$
\begin{aligned}
& \exists \tau \in{ }^{*} S_{n}: \bigwedge_{\ell<j} \bigwedge_{n_{1}<\cdots<n_{\ell}<j} \bigwedge_{f_{1}, \ldots, f_{\ell}, f, f^{\prime} \in \mathcal{F}} \\
& \left(f_{1}\left(x_{n_{1}}\right)+\cdots+f_{\ell}\left(x_{n_{\ell}}\right)+f(\tau) \text { is defined and in }{ }^{*} A\right. \\
& \left.\wedge f_{1}\left(x_{n_{1}}\right)+\cdots+f_{\ell}\left(x_{n_{\ell}}\right)+f(\tau)+{ }^{*} f^{\prime}\left(\alpha_{n}\right) \text { is defined and in }{ }^{* *} A\right)
\end{aligned}
$$

so by transfer, we get $x_{j} \in S_{n}$ with similar properties. Continue ad infinitum.

Theorem 46. Suppose $S$ is $\mathcal{F}$-Ramsey with $\delta$ layers. Then, for any finite colouring of $S$, there exists a block sequence $\left(x_{i}\right)_{i<\delta} \in \prod_{i<\delta} S_{i}$ such that for every $k<\delta$, the following set is monochromatic:

$$
S_{k} \cap\left\{f_{1}\left(x_{n_{1}}\right)+\cdots+f_{\ell}\left(x_{n_{\ell}}\right): 0 \leqslant n_{1}<\cdots<n_{\ell}<\delta, f_{1}, \ldots, f_{\ell} \in \mathcal{F}\right\}
$$

Proof. Identical to that of Theorem 45, but at each stage when defining $x_{i}$, replace $\alpha_{n}$ with $\alpha_{i}$, and $S_{n}$ with $S_{i}$. Continue up to stage $\delta$.

\footnotetext{
${ }^{10}$ This is a finite sentence, since $\mathcal{F}$ is locally finite by assumption.
} 
Theorem 45 easily implies a finite version:

Theorem 47. Suppose $S$ is $\mathcal{F}$-Ramsey with $\delta$ layers, and $n<\delta, m \in \mathbb{N}$. Then, for any finite colouring of $S$, there exists a block sequence $\left(x_{1}, \ldots, x_{m}\right) \subseteq S_{n}$ of length $m$ such that for every $k \leqslant n$, the following set is monochromatic:

$$
S_{k} \cap\left\{f_{1}\left(x_{n_{1}}\right)+\cdots+f_{\ell}\left(x_{n_{\ell}}\right): n_{1}<\cdots<n_{\ell} \leqslant m, f_{1}, \ldots, f_{\ell} \in \mathcal{F}\right\}
$$

Corollary $48(\boldsymbol{m}=\mathbf{1})$. Suppose $S$ is $\mathcal{F}$-Ramsey with $\delta$ layers, and $n<\delta$. Then, for any finite colouring of $S$, there exists $x \in S_{n}$ such that for every $k \leqslant n, S_{k} \cap\{f(x): f \in \mathcal{F}\}$ is monochromatic.

Incredibly, given the existence of any $\mathcal{F}$-coherent element in $\Pi_{S}$, we can construct an $\mathcal{F}$-Ramsey, and thus show that the general Ramsey statements above hold in $S$. The following lemma is essential to this construction.

Lemma 49. Suppose that $\left(\alpha_{i}\right)_{i<\delta} \in \Pi_{S}$ is $\mathcal{F}$-coherent and $u$-idempotent. Then, for any $f \in \mathcal{F}$ and $k<\delta$, there is $j=j_{k} \leqslant k$ such that

$$
f\left[\alpha_{k}+{ }^{*} \alpha_{k-1}+\cdots+{ }^{(k-1) *} \alpha_{1}+{ }^{k *} \alpha_{0}\right] \sim \alpha_{j}+{ }^{*} \alpha_{j-1}+\cdots+{ }^{(j-1) *} \alpha_{1}+{ }^{j *} \alpha_{0}
$$

Proof. By induction on $k$. Given $f \in \mathcal{F}$, the $\mathcal{F}$-coherence of $\left(\alpha_{i}\right)$ implies that $f\left(\alpha_{0}\right) \sim \alpha_{0}$. Thus, the base case $k=0$ holds with $j_{0}=0$. Now, suppose the lemma holds for $k>0$ we prove the $k+1$ case. By $\mathcal{F}$-coherence, there is $\ell \leqslant k+1$ such that $f\left(\alpha_{k+1}\right) \sim \alpha_{\ell}$, and Definition 35.(iii) and 35.(iv) imply that either $\ell=j_{k}$ or $\ell=j_{k}+1$.

Case 1: $\ell=j_{k}$. Then,

$$
\begin{aligned}
& f\left[\alpha_{k+1}+\left({ }^{*} \alpha_{k}+{ }^{* *} \alpha_{k-1}+\cdots+{ }^{k *} \alpha_{1}+{ }^{(k+1) *} \alpha_{0}\right)\right] \\
& \sim f\left(\alpha_{k+1}\right)+f\left[{ }^{*} \alpha_{k}+{ }^{* *} \alpha_{k-1}+\cdots+{ }^{k *} \alpha_{1}+{ }^{(k+1) *} \alpha_{0}\right] \quad f \text { homomorphism } \\
& \sim \alpha_{j}+{ }^{*} \alpha_{j}+{ }^{* *} \alpha_{j-1}+\cdots+{ }^{j *} \alpha_{1}+{ }^{(j+1) *} \alpha_{0} \\
& \sim \alpha_{j}+{ }^{*}\left({ }^{*} \alpha_{j-1}+\cdots+{ }^{(j-1) *} \alpha_{1}+{ }^{j *} \alpha_{0}\right) \\
& \sim \alpha_{j}+{ }^{*} \alpha_{j-1}+\cdots+{ }^{(j-1) *} \alpha_{1}+{ }^{j *} \alpha_{0} \quad \text { Proposition } 8
\end{aligned}
$$

Case 2: $\ell=j_{k}+1$. We can prove similarly that

$$
f\left[\alpha_{k+1}+{ }^{*} \alpha_{k}+\cdots+{ }^{k *} \alpha_{1}+{ }^{(k+1) *} \alpha_{0}\right] \sim \alpha_{j+1}+{ }^{*} \alpha_{j}+\cdots+{ }^{j *} \alpha_{1}+{ }^{(j+1) *} \alpha_{0}
$$

In either case, $j_{k+1}:=\ell$ is as required.

Theorem 50. Suppose $S$ has an $\mathcal{F}$-coherent element $\left(\alpha_{i}\right)_{i<\delta} \in \Pi$. Then, $S$ is $\mathcal{F}$-Ramsey.

Proof. The following argument is based on [20]. Recall that $\Pi$ is a compact $u$-semigroup by Proposition 34. We show that the subset $\Pi_{\mathcal{F}} \subseteq \Pi$ of $\mathcal{F}$-coherent elements is a closed $u$-subsemigroup. 
$\Pi_{\mathcal{F}}$ closed: apply Lemma 11, writing $\Pi_{\mathcal{F}}$ as

$$
\Pi_{\mathcal{F}}=\bigcap_{j<\delta} \bigcup_{k \leqslant j}\left\{\left(\alpha_{i}\right) \in \Pi: f\left(\alpha_{j}\right) \sim \alpha_{k}\right\}
$$

$\Pi_{\mathcal{F}} u$-subsemigroup: take $\left(\beta_{i}\right)_{i=0}^{\infty},\left(\gamma_{i}\right)_{i=0}^{\infty} \in \Pi_{\mathcal{F}}$. Then, $\left(\beta_{i}\right)+{ }^{*}\left(\gamma_{i}\right)=\left(\beta_{i}+{ }^{*} \gamma_{i}\right)$ is $\mathcal{F}$ coherent, by Proposition 8 and the fact that all $f \in \mathcal{F}$ are homomorphisms. By transfer, there exists $\left(\delta_{i}\right) \in \Pi_{\mathcal{F}}$ with $\left(\delta_{i}\right) \sim\left(\beta_{i}+{ }^{*} \gamma_{i}\right)$, as required.

It follows that $\Pi_{\mathcal{F}}$ is itself a compact $u$-semigroup. By induction on $k<\delta$, we will construct a sequence of elements $\left(\alpha_{i}^{(k)}\right)_{i<\delta} \in \Pi_{\mathcal{F}}$ with the following properties:

(i) For all $i \leqslant k, \alpha_{i}^{(k)} \sim \alpha_{i}^{(k-1)}$;

(ii) For all $i \in \mathbb{N}, \alpha_{i}^{(k)}$ is $u$-idempotent;

(iii) For all $i \leqslant k$ and $j \geqslant i$, we have $\alpha_{j}^{(k)}+{ }^{*} \alpha_{i}^{(k)} \sim \alpha_{j}^{(k)}$.

To begin, take any $u$-idempotent $\left(\alpha_{i}^{\prime}\right)_{i<\delta} \in \Pi_{\mathcal{F}}$ by Lemma 14. Let

$$
Z_{0}=\left\{\left(\beta_{i}\right)_{i<\delta} \in \Pi_{\mathcal{F}}: \beta_{0} \sim \alpha_{0}^{\prime}, \forall j \beta_{j}+{ }^{*} \alpha_{0}^{\prime} \sim \beta_{j}\right\}
$$

Then, $Z_{0}$ is a compact $u$-semigroup by Lemma 11 . Furthermore, it is nonempty, since it contains the element $\left(\beta_{i}\right)_{i<\delta}$ defined by

$$
\beta_{i} \sim \alpha_{i}^{\prime}+{ }^{*} \alpha_{0}^{\prime}
$$

To see this is in $\Pi_{\mathcal{F}}$, observe that for any $f \in \mathcal{F}, f\left[\alpha_{0}^{\prime}\right] \sim \alpha_{0}^{\prime}$, since $f$ is regressive and $\left(\alpha_{i}^{\prime}\right)$ is $\mathcal{F}$-coherent. Then we have

$$
f\left(\beta_{i}\right) \sim f\left[\alpha_{i}^{\prime}+{ }^{*} \alpha_{0}^{\prime}\right] \sim f\left[\alpha_{i}^{\prime}\right]+{ }^{*} f\left[\alpha_{0}^{\prime}\right] \sim \alpha_{j}^{\prime}+{ }^{*} \alpha_{0}^{\prime} \sim \beta_{j}
$$

for some $j \leqslant i$. It follows from the $u$-idempotence of $\alpha_{0}^{\prime}$ that $\left(\beta_{i}\right)_{i<\delta} \in Z_{0}$. Picking some $u$-idempotent $\left(\alpha_{i}^{(0)}\right)_{i<\delta} \in Z_{0}$, we can verify that $\left(\alpha_{i}^{(0)}\right)$ satisfies properties (i)-(iii) above with $k=0$.

Proceeding inductively, suppose sequences $\left(\alpha_{i}^{(0)}\right),\left(\alpha_{i}^{(1)}\right), \ldots,\left(\alpha_{i}^{(k-1)}\right)$ have been defined as required. Let

$$
Z_{k}=\left\{\left(\beta_{i}\right)_{i<\delta} \in \Pi_{\mathcal{F}}: \forall i \leqslant k\left(\beta_{i} \sim \alpha_{i}^{(k-1)} \wedge \forall j \geqslant i, \beta_{j}+{ }^{*} \alpha_{i}^{(k-1)} \sim \beta_{j}\right)\right\}
$$

Then, $Z_{k}$ is a compact $u$-semigroup by Lemma 11 . Furthermore, it is nonempty, since it contains the element $\left(\beta_{i}\right)_{i<\delta}$ defined by

$$
\beta_{i} \sim \alpha_{i}^{(k-1)}+{ }^{*} \alpha_{i-1}^{(k-1)}+{ }^{* *} \alpha_{i-2}^{(k-1)}+\cdots+{ }^{i *} \alpha_{0}^{(k-1)}
$$

which is $\mathcal{F}$-coherent by Lemma 49. Since $\left(\alpha_{i}^{(k-1)}\right)$ satisfies properties (i)-(iii), we have that $\left(\beta_{i}\right)_{i<\delta} \in Z_{k}$. Picking some $u$-idempotent $\left(\alpha_{i}^{(k)}\right)_{i<\delta} \in Z_{k}$, we can verify that $\left(\alpha_{i}^{(k)}\right)$ satisfies properties (i)-(iii) above. 
Finally, taking

$$
\alpha_{i} \sim \alpha_{0}^{0}+{ }^{*} \alpha_{1}^{1}+{ }^{* *} \alpha_{2}^{2}+\cdots+{ }^{i *} \alpha_{i}^{i}
$$

for each $i \in \mathbb{N}$, we get an $\mathcal{F}$-Ramsey sequence $\left(\alpha_{i}\right)_{i<\delta} \in \Pi_{\mathcal{F}}$.

Remark 51. In [11, Thm 3.8], assuming rather strong conditions on $S$ and $\mathcal{F}$, a direct construction of an $\mathcal{F}$-coherent is given. Along with Theorems 45 and 50, this gives sufficient conditions for a Ramsey statement on $S$ and $\mathcal{F}$ to be true. Unfortunately, such conditions do not apply to many examples of layered semigroups which we consider.

\section{Applications}

\subsection{Gowers' theorem}

Let FIN be the Gowers semigroup of Example 24, and $\mathcal{F}$ be the collection of generalised tetris operations on FIN, as in Example 39. For any $\varphi \in{ }^{\gamma} \mathrm{FIN}_{1}$, the sequence $\left(\alpha_{j}\right)$ defined $\alpha_{j}(n)=j \cdot \varphi(n)$ is $\mathcal{F}$-coherent. Thus, by Theorem 50, FIN is $\mathcal{F}$-Ramsey. Applying Theorem 45 gives us the generalised Gowers' theorem of Lupini [20, Thm 1.1]:

Corollary 52 (Lupini). For any finite colouring of FIN, there exists a block sequence $\left(f_{i}\right)_{i=1}^{\infty} \subseteq \mathrm{FIN}_{n}$ such that for every $k \leqslant n$, the following set is monochromatic:

$$
\mathrm{FIN}_{k} \cap\left\{\tilde{F}_{1}\left(f_{n_{1}}\right)+\cdots+\tilde{F}_{\ell}\left(f_{n_{\ell}}\right): n_{1}<\cdots<n_{\ell}, \tilde{F}_{1}, \ldots, \tilde{F}_{\ell} \in \mathcal{F}\right\}
$$

The finite version (due to Bartošová and Kwiatkowska [2, Cor 2.7]) is obtained from Theorem 47:

Corollary 53 (Bartošová-Kwiatkowska). For any $m \in \mathbb{N}$ and finite colouring of FIN, there exists a block sequence $\left(f_{1}, \ldots, f_{m}\right) \subseteq \mathrm{FIN}_{n}$ of length $m$ such that for every $k \leqslant n$, the following set is monochromatic:

$$
\mathrm{FIN}_{k} \cap\left\{\tilde{F}_{1}\left(f_{n_{1}}\right)+\cdots+\tilde{F}_{\ell}\left(f_{n_{\ell}}\right): n_{1}<\cdots<n_{\ell} \leqslant m, \tilde{F}_{1}, \ldots, \tilde{F}_{\ell} \in \mathcal{F}\right\}
$$

Gowers' original theorem [14, Theorem 1] is obtained by taking the subset $\mathcal{F}^{\prime} \subseteq \mathcal{F}$ consisting of iterates of the tetris operation $T(n)=\max \{n-1,0\}$. Since any $\mathcal{F}$-coherent sequence is also $\mathcal{F}^{\prime}$-coherent, it follows that FIN is $\mathcal{F}^{\prime}$-Ramsey, so Theorem 45 gives:

Corollary 54 (Gowers). For any finite colouring of FIN, there exists a block sequence $\left(f_{i}\right)_{i=1}^{\infty} \subseteq \mathrm{FIN}_{n}$ such that for every $k \leqslant n$, the following set is monochromatic:

$$
\mathrm{FIN}_{k} \cap\left\{\tilde{T}^{\left(m_{1}\right)}\left(f_{n_{1}}\right)+\cdots+\tilde{T}^{\left(m_{\ell}\right)}\left(f_{n_{\ell}}\right): n_{1}<\cdots<n_{\ell} ; m_{1}, \ldots, m_{\ell} \in \mathbb{N}\right\}
$$

where $\tilde{T}^{(m)}$ denotes the mth iterate of the tetris operation.

By identifying a set $A$ with its characteristic function $\mathbf{1}_{A}, \mathrm{FIN}_{1}$ is identified with $\mathcal{P}_{\text {fin }}(\mathbb{N})=\{A \subseteq \mathbb{N}: A$ finite $\}$. Then, the case $k=n=1$ gives Hindman's finite unions theorem [17, Cor 3.3]: 
Corollary 55 (Hindman). For any finite colouring of $\mathcal{P}_{\text {fin }}(\mathbb{N})$, there exists a disjoint sequence $\left(A_{i}\right)_{i=1}^{\infty}$ of finite subsets of $\mathbb{N}$ such that the set $\left\{A_{n_{1}} \cup \cdots \cup A_{n_{\ell}}: n_{1}<\cdots<n_{\ell}\right\}$ of all finite unions of elements of $\left(A_{i}\right)_{i=1}^{\infty}$ is monochromatic.

Gowers' theorem admits many variants. For $f, g \in$ FIN, say $f<g$ if $\max (\operatorname{supp}(f))<$ $\min (\operatorname{supp}(g))$. Then, we can further restrict the operation of pointwise sum $f+g$ to only be defined when $f<g$. The resulting semigroup is still adequate, and the ${ }^{\gamma} \mathrm{FIN}_{k}$ are identical to before. Therefore, exactly the same argument proves the strong Gowers' theorem:

Corollary 56 (Strong Gowers'). For any finite colouring of FIN, there exists an increasing sequence $\left(f_{1}<f_{2}<\cdots\right) \subseteq \mathrm{FIN}_{n}$ such that for every $k \leqslant n$, the following set is monochromatic:

$$
\mathrm{FIN}_{k} \cap\left\{\tilde{F}_{1}\left(f_{n_{1}}\right)+\cdots+\tilde{F}_{\ell}\left(f_{n_{\ell}}\right): n_{1}<\cdots<n_{\ell}, \tilde{F}_{1}, \ldots, \tilde{F}_{\ell} \in \mathcal{F}\right\}
$$

Again, $k=n=1$ gives a strengthening of Corollary 55-we can find an increasing sequence $\left(A_{1}<A_{2}<\cdots\right)$ with the same property, where for $A, B \subseteq \mathbb{N}, A<B \Longleftrightarrow$ $\max (A)<\min (B)$.

\subsection{The Graham-Rothschild theorem}

Let $W$ be the Graham-Rothschild semigroup of Example 21, and $\mathcal{F}$ be the collection of substitution maps $\tilde{w}$ on $W$, as in Example 38.

Theorem 57 ([4, 19]). There is an $\mathcal{F}$-coherent $\left(\alpha_{i}\right) \in \Pi_{W}$.

As we have seen, Theorem 57 is enough to deduce a Ramsey result about $W$. In fact, Theorems 45 and 46 give the main results from [4], infinitary versions of the GrahamRothschild theorem:

Corollary 58 (Carlson-Hindman-Strauss, Theorem 1.4). For any finite colouring of $W$, there exists a sequence $\left(u_{i}\right)_{i=0}^{\infty} \in \prod_{i=0}^{\infty} W_{i}$ such that for every $k \in \mathbb{N}$, the following set is monochromatic:

$$
W_{k} \cap\left\{u_{n_{1}}\left[\tilde{w}_{1}\right] \frown \ldots u_{n_{\ell}}\left[\tilde{w}_{\ell}\right]: n_{1}<\cdots<n_{\ell}, \tilde{w}_{1}, \ldots, \tilde{w}_{\ell} \in \mathcal{F}\right\}
$$

Corollary 59 (Carlson-Hindman-Strauss, Corollary 1.7). For any finite colouring of $W$, there exists a sequence $\left(u_{i}\right)_{i=0}^{\infty} \subseteq W_{n}$ such that for every $k \leqslant n$, the following set is monochromatic:

$$
W_{k} \cap\left\{u_{n_{1}}\left[\tilde{w}_{1}\right] \frown \ldots \frown u_{n_{\ell}}\left[\tilde{w}_{\ell}\right]: n_{1}<\cdots<n_{\ell}, \tilde{w}_{1}, \ldots, \tilde{w}_{\ell} \in \mathcal{F}\right\}
$$

Corollary 48 gives Graham and Rothschild's original theorem [15]:

Corollary 60 (Graham-Rothschild). For every $m \geqslant k$ and every finite colouring of $W_{k}$, there exists $u \in W_{m}$ such that the set $\left\{u[\tilde{w}]: \tilde{w} \uparrow_{m} \in W_{k}\right\}$ is monochromatic. 
The case $k=0, m=1$ of Corollary 60 gives the famed Hales-Jewett theorem:

Corollary 61 (Hales-Jewett). For every finite colouring of $W_{0}=A^{<\omega}$, there is a variable word $u \in W_{1}$ such that $\{u[a]: a \in A\}$ is monochromatic.

Corollary 61 is known to imply van der Waerden's theorem [16, p38]:

Corollary 62 (van der Waerden). For every $k \in \mathbb{N}$ and finite colouring of $\mathbb{N}$, there is $a, d \in \mathbb{N}$ such that the arithmetic progression $\{a+j d: 0 \leqslant j<k\}$ is monochromatic.

Remark 63. A construction satisfying Theorem 57 is given by Carlson, Hindman and Strauss [4, Thm 2.12], and by Hindman, Strauss and Zamboni [19]. In fact, in both cases, they directly construct an $\mathcal{F}$-Ramsey, all of whose elements are $\preccurlyeq$-minimal. Both arguments used to construct such a sequence are extraordinarily involved, long and complicated. The author believes that an $\mathcal{F}$-coherent could be constructed by a significantly simpler argument, from which an $\mathcal{F}$-Ramsey could be constructed via the general framework laid out in this paper, but has not been able to significantly simplify their argument, nor find an alternative method.

Remark 64. Nonetheless, if we restrict to the subset $\mathcal{F}^{\prime} \subseteq \mathcal{F}$ of strong variable words, i.e. those where all variables appear in order, a simple nonstandard argument can construct an $\mathcal{F}^{\prime}$-coherent. We rely on the following result from the theory of compact semitopological semigroups:

Lemma 65 ([24, Lemma 2.3]). If $\alpha \in{ }^{*} K$ is u-idempotent, and $I \subseteq{ }^{*} K$ is a closed left ideal, then there is u-idempotent $\beta \in I+{ }^{*} \alpha$ such that $\beta \preccurlyeq \alpha$.

Lemma 66 ([6, Lemma 7.9]). There are u-idempotents $\nu \in{ }^{*} W_{0}, \bar{\omega} \in{ }^{*} W_{1}$ such that $\bar{\omega} \preccurlyeq \nu$ and $\bar{\omega}[a] \sim \nu$ for every $a \in A$.

Proof. Let $T:=W_{0} \cup W_{1}$. Pick any $\preccurlyeq$-minimal $u$-idempotent $\nu \in{ }^{*} W_{0}$ by Lemma 16 . Note that ${ }^{*} W_{1}$ is a closed left ideal of ${ }^{*} T$, so by Lemma 65 , pick $u$-idempotent $\bar{\omega} \in$ ${ }^{*} W_{1} \frown{ }^{*} \nu \subseteq{ }^{*} W_{1}$ such that $\bar{\omega} \preccurlyeq \nu$. Now, for any $a \in A$, the substitution map $v \mapsto v[a]$ is a homomorphism $T \rightarrow W_{0}$, hence $\bar{\omega}[a] \preccurlyeq \nu[a]=\nu$ since $\nu \in{ }^{*} W_{0}$. Since $\nu$ is $\preccurlyeq$-minimal, it follows that $\bar{\omega}[a] \sim \nu$.

Theorem 67. There is an $\mathcal{F}^{\prime}$-coherent $\left(\alpha_{i}\right) \in \Pi_{W}$.

Proof. Let $\nu, \bar{\omega}$ be as in Lemma 66, and define $\alpha_{i}$ by $\alpha_{0}=\nu, \alpha_{1}=\bar{\omega}$, and

$$
\alpha_{i} \sim \bar{\omega}\left[x_{1}\right]^{\frown *} \bar{\omega}\left[x_{2}\right] \frown \ldots \frown i * \bar{\omega}\left[x_{i}\right]
$$

for $i \geqslant 2$. By $u$-idempotence of $\nu, \bar{\omega}$ and the fact that $\bar{\omega} \preccurlyeq \nu,\left(\alpha_{i}\right)$ is $\mathcal{F}^{\prime}$-coherent. 


\subsection{Galvin-Glazer and Hindman's theorem}

Let $S$ be an adequate partial semigroup such that $s+s$ is never defined. $S$ is trivially layered by the layering map $\ell: S \rightarrow \mathbb{N}, s \mapsto 0$. Let $\mathcal{F}$ consist of only the identity map id $: S \rightarrow S$, which is a regressive map. Then, any $\alpha \in{ }^{*} S$ is trivially $\mathcal{F}$-coherent, thus $S$ is $\mathcal{F}$-Ramsey. Applying Theorem 45 with $n=0$ gives the Galvin-Glazer theorem (see [24, Thm 2.20] or [5]):

Corollary 68 (Galvin-Glazer). For any finite colouring of $S$, there exists an infinite sequence $\left(x_{i}\right)_{i=0} \subseteq S$ of distinct elements such that the set

$$
\left\{x_{n_{0}}+\cdots+x_{n_{\ell-1}}: n_{0}<\cdots<n_{\ell-1},\right\}
$$

is c-monochromatic.

The special case $S=\mathbb{N}$ and $A=\left\{x_{i}: i \in \mathbb{N}\right\}$ gives us Hindman's finite sums theorem [17, Thm 3.1]:

Corollary 69 (Hindman). For any finite colouring $c: \mathbb{N} \rightarrow r$, there exists an infinite set $A \subseteq \mathbb{N}$, such that the set $\mathrm{FS}(A)$ of all finite, nonrepeating sums from $A$ is $c$ monochromatic.

\section{Located variable words}

In this section, we give a simple nonstandard proof of Bergelson, Blass and Hindman's partition theorem for located variable words [1, Thm 4.1], using Theorem 57 and the general framework of layered semigroups developed herein. In fact, we obtain a multivariable generalisation of that result, related to a result of Solecki [23, §4.3.1].

Fix a finite alphabet $A$. A located word over $A[21, \S 4.2]$ is a partial function $w: \mathbb{N} \rightarrow$ $A$, where $\operatorname{dom}(w)$ is finite and nonempty. In the vein of Example 21, a located $k$-parameter word over $A$ is a partial function $w: \mathbb{N} \rightarrow A \cup\left\{x_{1}, \ldots, x_{k}\right\}$, where $\operatorname{dom}(w)$ is finite, all the $x_{i}$ appear, and in increasing order.

For each $k \in \mathbb{N}$, we let $L_{k}$ be the set of all located $k$-parameter words over $A$ (where $L_{0}$ simply contains located words). Then, $L=\bigcup_{i=0}^{\infty} L_{i}$ is a layered semigroup, with the operation + defined

$$
(w+v)(n)= \begin{cases}w(n) & \text { if } n \in \operatorname{dom}(w) \\ v(n) & \text { if } n \in \operatorname{dom}(v) \\ \text { undefined } & \text { otherwise }\end{cases}
$$

only when $\operatorname{dom}(w) \cap \operatorname{dom}(v)=\varnothing$. Notice that $L$ is an adequate partial semigroup, and the ${ }^{\gamma} L_{m}$ are exactly the cofinite located $m$-variable words $\alpha$-those whose domains $\operatorname{dom}(\alpha) \subseteq{ }^{*} \mathbb{N}$ are disjoint from $\mathbb{N}$. 
As in Example 38, substitution maps $u \mapsto u[\tilde{w}]$ are defined by infinite (total/nonlocated) variable words $\tilde{w}$, in much the same way:

$$
u[\tilde{w}](n)= \begin{cases}u(n) & \text { if } n \in \operatorname{dom}(u), u(n) \neq x_{i} \text { for all } i \\ \tilde{w}_{i} & \text { if } n \in \operatorname{dom}(u), u(n)=x_{i} \text { for some } i \\ \text { undefined } & \text { if } n \notin \operatorname{dom}(u)\end{cases}
$$

In fact, we can naturally identify the Graham-Rothschild semigroup $W$ as a subset of $L$, and the above definition extends the substitution maps on $W$. As before, all such substitution maps are regressive, and the collection $\mathcal{F}$ of all of them is locally finite, and closed under composition.

Assuming the existence of an $\mathcal{F}$-coherent in $\Pi_{W}$, we can construct an $\mathcal{F}$-coherent in $\Pi_{L}$ by a simple nonstandard argument:

Lemma 70. L has an $\mathcal{F}$-coherent.

Proof. Pick an $\mathcal{F}_{W}$-coherent sequence $\left(\alpha_{i}\right)_{i=0}^{\infty}$ in the Graham-Rothschild semigroup. Note that all substitution maps preserve the length of words, thus each $\alpha_{i}$ must have infinite length. If any $\alpha_{i}$ was finite, $\mathcal{F}$-coherence and Proposition 8.(i) would imply all the $\alpha_{i}$ are finite, and of the same length $\left|\alpha_{i}\right|=n$. But then $\alpha_{n+1}$ cannot exist, since there are no $(n+1)$-parameter words of length $n$.

Define $g: L \rightarrow L$ as the map which takes the "second half" of any located variable word:

$$
g(w)(n)= \begin{cases}w(n) & n \geqslant\lfloor(|w| / 2)\rfloor \\ \text { undefined } & n<\lfloor(|w| / 2)\rfloor\end{cases}
$$

Note that $g$ commutes with every substitution map, i.e. $g(u[\tilde{w}])=g(u)[\tilde{w}]$ for all $u \in L$, $\tilde{w} \in \mathcal{F}$. By transfer, this is also true for nonstandard words $\alpha \in{ }^{*} L$.

Now, define $\beta_{i}=g\left(\alpha_{i}\right)$ for each $i \in \mathbb{N}$. Since the lengths $\left|\alpha_{i}\right| \in{ }^{*} \mathbb{N} \backslash \mathbb{N}$ are infinite, it follows that each $\beta_{i}$ is undefined up to $\left\lfloor\left(\left|\alpha_{i}\right| / 2\right)\right\rfloor \in{ }^{*} \mathbb{N} \backslash \mathbb{N}$, whence $\beta_{i} \in{ }^{\gamma} L_{i}$. Thus, $\left(\beta_{i}\right)_{i=0}^{\infty} \in \Pi_{L}$. The $\mathcal{F}$-coherence of $\left(\beta_{i}\right)$ follows from Proposition 8.(ii)-for all $i \in \mathbb{N}$ and $\tilde{w} \in \mathcal{F}$, there is $j \leqslant i$ such that

$$
\beta_{i}[\tilde{w}]=g\left(\alpha_{i}\right)[\tilde{w}]=g\left(\alpha_{i}[\tilde{w}]\right) \sim g\left(\alpha_{j}\right)=\beta_{j}
$$

It follows by Theorem 50 that $L$ is $\mathcal{F}$-Ramsey. Applying Theorems 45 gives a multivariable generalisation of the Bergelson-Blass-Hindman theorem on located words $[1$, Thm 4.1]:

Corollary 71. For any $n$ and finite colouring of $L$, there exists a block sequence $\left(u_{i}\right)_{i=1}^{\infty} \subseteq$ $L_{n}$ such that for every $k \leqslant n$, the following set is monochromatic:

$$
L_{k} \cap\left\{u_{n_{1}}\left[\tilde{w}_{1}\right]+\cdots+u_{n_{\ell}}\left[\tilde{w}_{\ell}\right]: n_{1}<\cdots<n_{\ell}, \tilde{w}_{1}, \ldots, \tilde{w}_{\ell} \in \mathcal{F}\right\}
$$

The original theorem of Bergelson, Blass and Hindman is the case $n=1$. If we instead take Corollary 71 with $n=k=1$ and $A=\varnothing$, we again obtain Hindman's finite unions theorem (Corollary 55). 
Remark 72. The proof of Lemma 70 is also valid for the subcollection $\mathcal{F}^{\prime} \subseteq \mathcal{F}$ of substitution maps corresponding to strong variable words (see Remark 64), so along with Theorem 67, this gives a short nonstandard proof of the weaker version of Corollary 71 for strong variable words.

Remark 73. As with Corollary 56, we can also restrict the operation $w+v$ to only be defined when $w<v$, i.e. $\operatorname{dom}(w)<\operatorname{dom}(v)$. The proof then goes through unchanged, and we get a stronger version of Corollary 71, where the block sequence $\left(u_{1}<u_{2}<\cdots\right) \subseteq L_{n}$ can be taken to be increasing.

\subsection{A common generalisation}

It is notable that Gowers' theorem and the Bergelson-Blass-Hindman theorem [1, Thm 4.1] both generalise the finite unions theorem of Hindman [17, Cor 3.3]. Here, we present a common generalisation of both theorems [20,21], and prove it using the general framework of layered semigroups previously described.

Let $A$ be a finite alphabet, and $X=\left\{x_{0}, x_{1}, x_{2}, \ldots\right\}$ be a countably infinite set of variables. Note that, compared to the Graham-Rothschild case, we have added an extra variable symbol $x_{0}$, which we interpret to mean "undefined". Let FIN $^{A}$ be the set of (total) $f: \mathbb{N} \rightarrow A \cup X$ which are eventually constant and equal to $x_{0}$.

For $f, g \in \mathrm{FIN}^{A}$, the sum $f+g$ is defined iff for every $n \in \mathbb{N}$, at least one of $f(n), g(n)$ is $x_{0}$. In this case, $f+g$ is defined as

$$
(f+g)(n)= \begin{cases}f(n) & \text { if } f(n) \neq x_{0} \\ g(n) & \text { if } f(n)=x_{0}\end{cases}
$$

Under this operation, and the layering $\operatorname{map} \ell(f)=\max \left\{k: f(n)=x_{k}\right.$ for some $\left.n\right\}, \mathrm{FIN}^{A}$ is a commutative, adequate, partial layered semigroup.

Call a (total) map $F: \mathbb{N} \rightarrow A \cup X$ strong if $F(0)=x_{0}$, and all $x_{i}$ appear as values of $F$ in increasing order (i.e. whenever $F(n)=x_{i}, F(m)=x_{j}$ for $n \leqslant m$, then $i \leqslant j$ ). The strong maps act on FIN $^{A}$ by "composition":

$$
\tilde{F}[f](n)= \begin{cases}F(k) & \text { if } f(n)=x_{k} \text { for some } k \geqslant 1 \\ f(n) & \text { if } f(n) \in A \cup\left\{x_{0}\right\}\end{cases}
$$

Every strong $\tilde{F}$ is a regressive map, and the collection $\mathcal{F}$ of all such strong maps is locally finite, and closed under composition.

Identifying "undefined" with the symbol $x_{0}$, the semigroup $L^{A}$ of located words over $A$ is a subset of $\mathrm{FIN}^{A}$. As this containment is strict, we need to strengthen Theorem 45 to ensure that the sequence can be found inside a specified subsemigroup $T \subseteq \mathrm{FIN}^{A}$. Henceforth, we assume $T$ is layerwise adequate, and closed under all strong maps. We define $\Pi_{T}:=\prod_{i=0}^{\infty} \gamma T_{i}$, and say that $T$ is $\mathcal{F}$-Ramsey if $\Pi_{T}$ contains an $\mathcal{F}$-Ramsey element. Theorem 45 admits the following strengthening: 
Theorem 74. Suppose $T \subseteq \mathrm{FIN}^{A}$ is $\mathcal{F}$-Ramsey, and $n \in \mathbb{N}$. Then, for any finite colouring of $\mathrm{FIN}^{A}$, there exists a block sequence $\left(f_{i}\right)_{i=1}^{\infty} \subseteq T_{n}:=T \cap \mathrm{FIN}_{n}^{A}$ such that for every $k \leqslant n$, the following set is monochromatic:

$$
\operatorname{FIN}_{k}^{A} \cap\left\{\tilde{F}_{1}\left(f_{n_{1}}\right)+\cdots+\tilde{F}_{\ell}\left(f_{n_{\ell}}\right): n_{1}<\cdots<n_{\ell}, \tilde{F}_{1}, \ldots, \tilde{F}_{\ell} \in \mathcal{F}\right\}
$$

Proof. As for Theorem 45, but replace all occurrences of $S_{n}$ with $T_{n}$.

The question remains - when is $T \mathcal{F}$-Ramsey?

Definition 75. A subsemigroup $T \subseteq \mathrm{FIN}^{A}$ is complete if for any $\alpha \in{ }^{\gamma} T_{1}$ and $k \in \mathbb{N}$, we have $\alpha+{ }^{*} \alpha\left[x_{2}\right]+\cdots+{ }^{(k-1) *} \alpha\left[x_{k}\right] \in{ }^{k *} T$, where $\alpha[b]$ is the function obtained by setting $\alpha[b](n)=b$ whenever $\alpha(n)=x_{1}$.

Lemma 76. Every complete $T \subseteq \mathrm{FIN}^{A}$ is $\mathcal{F}$-Ramsey.

Proof. Pick $u$-idempotent $\nu \in{ }^{\gamma} T_{0}, \bar{\omega} \in{ }^{\gamma} T_{1}$ such that $\bar{\omega} \preccurlyeq \nu$ and $\bar{\omega}[a] \sim \nu$ for every $a \in A$ (see Lemma 66). Since $T$ is complete, the argument in Theorem 67 works, giving an $\mathcal{F}$-coherent in $\Pi_{T}$. Theorem 50 now shows $T$ is $\mathcal{F}$-Ramsey, by restricting to $\Pi_{T}$ rather than $\Pi_{S}$.

Note that FIN $^{A}$ is trivially complete, hence it is $\mathcal{F}$-Ramsey. Applying Theorem 74 with $A=\varnothing$ and $T=$ FIN $^{\varnothing}=$ FIN gives Gowers' theorem (Corollary 52, where we interpret each variable symbol $x_{k}$ as the number $\left.k \in \mathbb{N}\right) . L_{A} \subseteq \mathrm{FIN}^{A}$ is also complete, and Theorem 74 with $T=L_{A}$ gives the Bergelson-Blass-Hindman theorem for strong variable words (Corollary 71 , interpreting $x_{0}$ to mean "undefined").

Remark 77. Theorem 74 doesn't recover the full strength of Corollary $71-$ we could attempt to do so by considering a wider class $\mathcal{F}^{+}$of regressive maps $F: \mathbb{N} \rightarrow A \cup X$, where only the first occurrences of each $x_{i}$ must appear in increasing order. It is unclear whether FIN $^{A}$ is $\mathcal{F}^{+}$-Ramsey. If it were, we would expect that the argument to construct an $\mathcal{F}^{+}$-coherent would be more complicated, as per Remark 63 . This would give an even more generalised version of Gowers' theorem, where some layers of a function $f \in$ FIN can be "reversed".

In the same way as Lupini $[20, \S 3]$ and Dodos-Panellopoulos [8, Thm 2.21], we can generalise Theorem 74 to cover the Milliken-Taylor theorem. For $m \in \mathbb{N}$, let $\operatorname{FIN}^{A[m]}$ be the collection of block sequences $\left(f_{1}, \ldots, f_{m}\right)$ of elements of FIN $^{A}$.

Theorem 78. Suppose $T \subseteq \mathrm{FIN}^{A}$ is $\mathcal{F}$-Ramsey, and $n \in \mathbb{N}$. Then, for any finite colouring of $\mathrm{FIN}^{A}$, there exists a block sequence $\left(x_{i}\right)_{i=1}^{\infty} \subseteq T_{n}$ such that for every $k \leqslant n$, the following set is monochromatic:

$$
\begin{aligned}
& \left\{\left(\tilde{F}_{1}\left(x_{n_{1}}\right)+\cdots+\tilde{F}_{\ell_{1}}\left(x_{n_{\ell_{1}}}\right), \ldots, \tilde{F}_{\ell_{m-1}+1}\left(x_{n_{\ell_{m-1}+1}}\right)+\cdots+\tilde{F}_{\ell_{m}}\left(x_{n_{\ell_{m}}}\right)\right):\right. \\
& \left.0<\ell_{1}<\cdots<\ell_{m} ; n_{1}<\cdots<n_{\ell_{m}} ; \tilde{F}_{1}, \ldots, \tilde{F}_{\ell_{m}} \in \mathcal{F}\right\} \cap \operatorname{FIN}_{k}^{A[m]}
\end{aligned}
$$

Proof. Identical to [6, Thm 8.16].

Theorem 74 is the case $m=1$, while the Milliken-Taylor theorem is the case $A=\varnothing$, $T=$ FIN $^{\varnothing}=$ FIN,$n=1$. 


\section{Closing remarks}

This paper outlines a general framework for proving Ramsey-type results about layered semigroups. We have seen in $\S 6$ and $\S 7$ that many old results in Ramsey theory can be expressed and proven via our framework. Since layered semigroups form a rich class of structures, we also expect that one could use this framework to prove new Ramseytheoretic results. Unfortunately, we are not aware of any immediate applications to known open problems.

Problem 79. Use the framework of this paper to prove new Ramsey-theoretic results of interest.

We note that there are still Ramsey statements about semigroups which we have not been able to recover. In particular, we saw that van der Waerden's theorem is a corollary of the Graham-Rothschild theorem, which can be proven in our framework, but a direct formulation remains elusive. We could attempt to obtain it from Theorem 46 by letting $S=\mathbb{N}^{+} \times\{0,1\}$ and $\ell:(n, i) \mapsto i$. Then, consider maps $f_{c}: S \rightarrow S_{0}$, where $f_{c}:(n, 0) \mapsto(n, 0),(m, 1) \mapsto(c m, 0)$ for all $c$ up to some fixed $k$. Taking the operation + on $S_{0}$ to be usual addition, Theorem 46 obtains the structure of van der Waerden's theorem. However, it may not be possible to extend + to $S$ so that $(S, \ell)$ is layered and the $f_{c}$ are homomorphisms.

Question 80. Is there a layered semigroup $S$, and regressive maps $\mathcal{F}$ on $S$, such that Theorem 45 or Theorem 46 reduce to van der Waerden's theorem?

Another result notably missing is Ramsey's theorem itself. The most sensible attempt seems to be taking $S=\mathcal{P}_{\text {fin }}(\mathbb{N})=\{F \subseteq \mathbb{N}: F$ finite $\}$, and layering it by $\ell: F \mapsto|F|$. Then, given a sequence $\left(F_{i}\right)_{i=1}^{\infty}$ of finite sets, an infinite homogeneous set $B$ could be obtained by $B=\bigcup_{i=1}^{\infty} F_{i}$. The challenge is how to define a semigroup operation and regressive maps that allow us to generate any finite subset of $B$.

Question 81. Is there a layered semigroup $S$, and regressive maps $\mathcal{F}$ on $S$, such that Theorem 45 or Theorem 46 reduce to Ramsey's theorem?

Definition 35 imposed strong conditions on the types of maps considered, particularly conditions (iii) and (iv). While all examples considered satisfied all these conditions, perhaps a different argument is possible which doesn't require these conditions. This would require a new construction in the proof, since conditions (iii) and (iv) are necessary to Lemma 49.

Question 82. Can Theorem 50 be proven when conditions (iii) and/or (iv) are weakened or removed from Definition 35?

Our framework allows us to deduce Ramsey statements about $(S, \mathcal{F})$ from the existence of an $\mathcal{F}$-coherent in $S$. However, we have not found a general way to construct $\mathcal{F}$-coherents in arbitrary layered semigroups $S$, or ensure they exist. The most general construction we 
have given of an $\mathcal{F}$-coherent is Lemma 76 , but this still depends crucially on the structure of $\mathrm{FIN}^{A}$. Farah-Hindman-McLeod [11, Thm 3.8] give a fairly general construction, but it requires very strong conditions on $\mathcal{F}$, which do not hold in many natural examples.

Problem 83. Find a general way to construct an $\mathcal{F}$-coherent in a layered semigroup $S$, making as few assumptions about $S$ and $\mathcal{F}$ as possible.

We did not present a construction of an $\mathcal{F}$-coherent in the Graham-Rothschild semigroup $W$, instead deferring to a result of Carlson-Hindman-Strauss [4, Thm 2.12]. They directly construct a $\preccurlyeq$-minimal $\mathcal{F}$-Ramsey, and as a result, their argument is extremely complicated. Morally, if we only need to construct an $\mathcal{F}$-coherent, there should be a simpler argument - then our framework would imply the Graham-Rothschild theorem.

Problem 84. Find a simpler construction of an $\mathcal{F}$-coherent in the Graham-Rothschild semigroup $W$.

Our results generalise many Ramsey-type or partition results on layered semigroups. However, there is another thread of Ramsey theory dealing with density results, having the form that any set $A \subseteq \mathcal{M}$ of positive "density" contains a substructure $\mathcal{N} \subseteq \mathcal{M}$ with certain properties. Often, we take $\mathcal{M}=\mathbb{N}$ and interpret density to mean upper density; $d(A)=\limsup _{n \rightarrow \infty}|A \cap[0, n)| / n$ for each $A \subseteq N$. For example, Szemerédi's theorem is the density version of van der Waerden's theorem:

Theorem 85 (Szemerédi). For any $k \in \mathbb{N}$ and $A \subseteq \mathbb{N}$ with $d(A)>0$, there are $a, d \in \mathbb{N}^{+}$ such that the arithmetic progression $\{a+c d: 0 \leqslant c<k\} \subseteq A$.

By defining a suitable notion of density on $W$, Furstenberg and Katznelson obtained a density version of the Hales-Jewett theorem [12]. Nonstandard methods have also been applied successfully to prove density-type results [6, Part III]. Thus, it may be possible to develop a similar framework for proving density theorems in layered semigroups. This would require a suitable notion of density on layered semigroups-McLeod has already generalised some combinatorial notions of size in $\mathbb{N}$ to this setting [22].

Problem 86. Develop an analogous framework for proving density results on layered semigroups.

Variations on the basic structure of Theorem 45 also appear in Ramsey theory. For example, take the Graham-Rothschild semigroup $W$, and let $L V \subseteq W$ be the subset consisting of left-variable words - those whose first character is the variable $x_{1}$. The Hales-Jewett theorem for left-variable words states:

Theorem 87 ([24, Theorem 2.37]). For any finite colouring of $W_{0}=A^{<\omega}$, there exists a word $w \in W_{0}$ and a sequence $\left(u_{i}\right)_{i=0}^{\infty} \subseteq L V \cap W_{1}$ of left-variable words such that the following set is monochromatic:

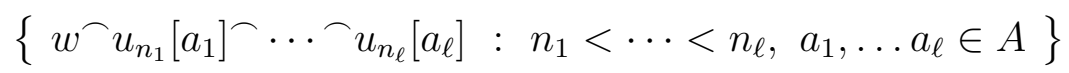


Effectively, we ensure that all products of subsequences have the same nonvariable part. $L V \subseteq W$ is a right ideal, so we could generalise this idea to right layered semigroups $(S, \ell, R)$, with $R \subseteq S$ a distinguished right ideal meeting every layer except $S_{0}$. Then, we instead consider sequences in $\Lambda_{S}:={ }^{*} S_{0} \times \prod_{1 \leqslant i<\delta}{ }^{*} R_{i}$, and define analogous notions of right $\mathcal{F}$-coherent and right $\mathcal{F}$-Ramsey sequences. Further investigation is required to see if the proofs of Theorems 45 and 50 translate to this setting. Problems may arise in translating the proof of Theorem 50, if we require conditions such as $\kappa+{ }^{*} f\left(\alpha_{i}\right) \sim \kappa+{ }^{*} \alpha_{j}$, which are not continuous in $\alpha_{i}$.

Problem 88. Translate our ideas to the setting of right layered semigroups.

\section{Acknowledgements}

Thanks go to:

- Martino Lupini, for teaching me all I know about Ramsey theory and nonstandard analysis, and for first noticing the abstract connection between Gowers' theorem and the Graham-Rothschild theorem, eventually leading to the ideas in this paper.

- Valentino Vito, for thoroughly reading the first draft, and providing invaluable suggestions and corrections.

- The anonymous reviewer, for their thorough peer review and useful comments.

\section{References}

[1] Vitaly Bergelson, Andreas Blass, and Neil Hindman. Partition theorems for spaces of variable words. Proceedings of the London Mathematical Society s3-68(3), pp. 449-476, 1994.

[2] Dana Bartošová and Aleksandra Kwiatkowska. Gowers' Ramsey theorem with multiple operations and dynamics of the homeomorphism group of the Lelek fan. Journal of Combinatorial Theory, Series A 150, pp. 108-136, 2017.

[3] Jordan Mitchell Barrett, Martino Lupini, and Joel Moreira. On Rado conditions for nonlinear Diophantine equations. European Journal of Combinatorics 94C, \#103277, 2021.

[4] Timothy J. Carlson, Neil Hindman, and Dona Strauss. An infinitary extension of the Graham-Rothschild parameter sets theorem. Transactions of the American Mathematical Society 358(7), pp. 3239-3262, 2006.

[5] W. W. Comfort. Ultrafilters: some old and some new results. Bulletin of the American Mathematical Society 83(4), pp. 417-455, 1977.

[6] Mauro Di Nasso, Isaac Goldbring, and Martino Lupini. Nonstandard Methods in Ramsey Theory and Combinatorial Number Theory. Lecture Notes in Mathematics 2239, Springer, 2019. 
[7] Mauro Di Nasso. Hypernatural numbers as ultrafilters. In: Peter A. Loeb and Manfred P. H. Wolff (eds.), Nonstandard Analysis for the Working Mathematician. Springer, 2015.

[8] Pandelis Dodos and Vassilis Kanellopoulos. Ramsey Theory for Product Spaces. Mathematical Surveys and Monographs 212, American Mathematical Society, 2016.

[9] Mauro Di Nasso and Lorenzo Luperi Baglini. Ramsey properties of nonlinear Diophantine equations. Advances in Mathematics 324, pp. 84-117, 2018.

[10] Mauro Di Nasso and Maria Riggio. Fermat-like equations that are not partition regular. Combinatorica 38(5), pp. 1067-1078, 2018.

[11] Ilijas Farah, Neil Hindman, and Jillian McLeod. Partition theorems for layered partial semigroups. Journal of Combinatorial Theory, Series A 98(2), pp. 268-311, 2002.

[12] H. Furstenberg and Y. Katznelson. A density version of the Hales-Jewett theorem. Journal d'Analyse Mathématique 57(1), pp. 64-119, 1991.

[13] V Farmaki and S Negrepontis. Ramsey theory with mixed types of substitution. http: //users.uoa.gr/ vfarmaki/CV25.pdf

[14] W. T. Gowers. Lipschitz functions on classical spaces. European Journal of Combinatorics 13(3), pp. 141-151, 1992.

[15] R. L. Graham and B. L. Rothschild. Ramsey's theorem for n-parameter sets. Transactions of the American Mathematical Society 159, pp. 257-292, 1971.

[16] Ronald L. Graham, Bruce L. Rothschild, and Joel H. Spencer. Ramsey Theory. 2nd ed. Wiley, 1990.

[17] Neil Hindman. Finite sums from sequences within cells of a partition of $\mathbb{N}$. Journal of Combinatorial Theory, Series A 17(1), pp. 1-11, 1974.

[18] Neil Hindman and Dona Strauss. Algebra in the Stone-Čech Compactification: Theory and Applications. 2nd ed. De Gruyter, 2012.

[19] Neil Hindman, Dona Strauss, and Luca Q. Zamboni. Combining extensions of the Hales-Jewett theorem with Ramsey theory in other structures. The Electronic Journal of Combinatorics 26(4), \#P4.23, 2019.

[20] Martino Lupini. Gowers' Ramsey theorem for generalized tetris operations. Journal of Combinatorial Theory, Series A 149, pp. 101-114, 2017.

[21] Martino Lupini. Actions on semigroups and an infinitary Gowers-Hales-Jewett Ramsey theorem. Transactions of the American Mathematical Society 371(5), pp. 30833116, 2019.

[22] Jillian McLeod. Some notions of size in partial semigroups. Topology Proceedings 25, pp. 317-332, 2000.

[23] Sławomir Solecki. Monoid actions and ultrafilter methods in Ramsey theory. Forum of Mathematics Sigma 7, e2, 2019.

[24] Stevo Todorčević. Introduction to Ramsey Spaces. Annals of Mathematics Studies 174, Princeton University Press, 2010. 